\title{
Discovery of a highly potent glucocorticoid for asthma treatment
}

Yuanzheng $\mathrm{He}^{1}$, Jingjing Shi ${ }^{2}$, Wei $\mathrm{Yi}^{2}$, Xin Ren ${ }^{3}$, Xiang Gao ${ }^{1}$, Jianshuang $\mathrm{Li}^{4,5}$, Nanyan $\mathrm{Wu}^{3}$, Kevin Weaver ${ }^{4}$, Qian Xie ${ }^{6}$, Sok Kean Khoo, Tao Yang ${ }^{4}$, Xiaozhu Huang ${ }^{3}$, Karsten Melcher ${ }^{1}$, H Eric Xu ${ }^{1,2}$

${ }^{1}$ Laboratory of Structural Sciences, Van Andel Research Institute, 333 Bostwick Avenue Northeast, Grand Rapids, MI, USA; ${ }^{2}$ VARI-SIMM Center, Center for Structure and Function of Drug Targets, Key Laboratory of Receptor Research, Shanghai Institute of Materia Medica, Chinese Academy of Sciences, Shanghai, China; ${ }^{3}$ Department of Medicine, Lung Biology Center, University of California, San Francisco, San Francisco, CA, USA; ${ }^{4}$ Laboratory of Skeletal Biology, Van Andel Research Institute, Grand Rapids, MI, USA; ${ }^{5}$ College of Life Sciences, Wuhan University, Wuhan, Hubei, China; ${ }^{6}$ Molecular Oncogenesis and Targeted Therapy, Laboratory of Molecular Oncology, Center of Cancer and Cell Biology, Van Andel Research Institute, Grand Rapids, MI, USA; ${ }^{7}$ Department of Cell and Molecular Biology, Grand Valley State University, Grand Rapids, MI, USA

Cell Discovery (2016) 2, 15051; doi:10.1038/celldisc.2015.51; published online 26 January 2016

Correction to: Cell Discovery (2015) 1, 15035; doi:10.1038/celldisc.2015.35; published online 15 December 2015

In the initial published version of this article, an error was made when we drew the chemical structure of VSGC12 (Figure 1a), a double bond is missing at the C-6 to C-7 position. Upon careful examination of our original chemical data, we found out that there should be a double bond at the C-6 to C-7 position, instead of a single bond in the initial Figure 1a (see below figure). We would also like to slightly change the description of the chemical structure of VSGC12 accordingly from 'Incorporating these modifications, as well as an F atom at the C-6 position, to VSG22 created a novel glucocorticoid, VSGC12 (Figure 1a)' (page 2 right panel lines 42-45) to 'Incorporating these modifications, as well as an $\mathrm{F}$ atom at the C-6 position and a double bond at C-6 to C-7 position, to VSG22 created a novel glucocorticoid, VSGC12 (Figure 1a)'. All figures and text description other than this remain the same as initially published. The unintentional mistake of the missing double bond at the C-6 to C-7 position in the chemical structure drawing does not affect the results and the conclusion of the paper. We sincerely apologize for any inconvenience that may have been caused by our error.

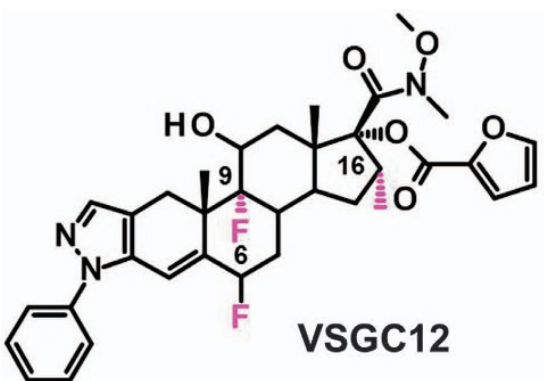

Original one

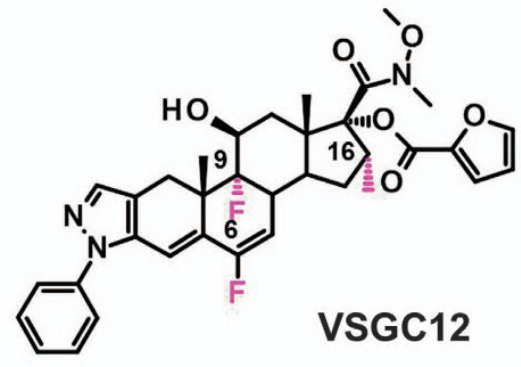

Corrected one

Figure 1 